Western University

Scholarship@Western

2013

\title{
Orientation Distribution of Highly Oriented Type I Collagen Deposited on Flat Samples with Different Geometries
}

\author{
Qamrun Nahar \\ Western University \\ David Minh Luan Quach \\ Western University \\ Behafarid Darvish \\ Western University \\ Harvey A. Goldberg \\ Western University \\ Bernd Grohe \\ Western University \\ See next page for additional authors
}

Follow this and additional works at: https://ir.lib.uwo.ca/physicspub

Part of the Astrophysics and Astronomy Commons, and the Physics Commons

Citation of this paper:

Nahar, Qamrun; Quach, David Minh Luan; Darvish, Behafarid; Goldberg, Harvey A.; Grohe, Bernd; and Mittler, Silvia, "Orientation Distribution of Highly Oriented Type I Collagen Deposited on Flat Samples with Different Geometries" (2013). Physics and Astronomy Publications. 45.

https://ir.lib.uwo.ca/physicspub/45 
Authors

Qamrun Nahar, David Minh Luan Quach, Behafarid Darvish, Harvey A. Goldberg, Bernd Grohe, and Silvia Mittler 
Langmuir 29(22) (2013) 6680-6686

\section{Orientation Distribution of Highly Oriented Type I}

\section{Collagen Deposited on Flat Samples with Different}

\section{Geometries}

Qamrun Nahar, ${ }^{\dagger \ddagger}$ David Minh Luan Quach $^{\dagger, \perp}$, Behafarid Darvish ${ }^{\dagger \S}$, Harvey A. Goldberg $\|, \uparrow$, Bernd Grohe, ${ }^{\Uparrow}$ and Silvia Mittler ${ }^{\dagger} * *$

${ }^{\dagger}$ Department of Physics and Astronomy, The University of Western Ontario (Western University), London, Ontario, Canada, N6A $3 K 7$

Department of Electrical and Computer Engineering, The University of Western Ontario (Western University), London, Ontario, Canada, N6A $5 B 9$

${ }^{\perp}$ Department of Chemistry, The University of Western Ontario (Western University), London, Ontario, Canada, N6A 5B7

$\S$ Biomedical Engineering, The University of Western Ontario (Western University), London, Ontario, Canada, N6A $5 B 9$

\| Department of Biochemistry, Schulich School of Medicine \& Dentistry, The University of Western Ontario (Western University), London, Ontario, Canada, N6A 5C1

"School of Dentistry, Schulich School of Medicine \& Dentistry, The University of Western Ontario (Western University), London, Ontario, Canada, N6A 5C1 
* Corresponding author: Silvia Mittler

Department of Physics and Astronomy

The University of Western Ontario (Western University)

London, ON, N6A 3K7

Canada

E-mail: smittler@uwo.ca

Phone: 1 (519) 661-2111 ext. 88592

Fax: 1 (519) 661-2033 


\begin{abstract}
The structural arrangement of type I collagen in vivo is critical for the normal functioning of tissues, such as bone, cornea, tendons and blood vessels. At present, there are no established low-cost techniques for fabricating aligned collagen structures for applications in regenerative medicine. Here, we report on a straightforward approach to fabricate collagen films, with defined orientation distributions of collagen fibrillar aggregates within a matrix of oriented collagen molecules on flat sample surfaces. Langmuir Blodgett (LB) technology was used to deposit thin films of oriented type I collagen onto flat substrates exhibiting various shapes. By varying the shapes of the substrates (e.g. rectangles, squares, circles, parallelograms, and various shaped triangles) as well as their sizes, a systematic study on collagen alignment patterns was conducted. It was found that the orientation and the orientation distribution of collagen along these various shaped substrates is directly depending on the geometry of the substrate and the dipping direction of that sample with respect to the collagen/water subphase.
\end{abstract}

An important factor in tissue engineering is the stability, durability and endurance of the constructed artificial tissue, and thus its functioning in regenerative medicine applications. By testing these criteria we found that the coated films and their alignments were stable for at least three months under different conditions and, moreover, that these films can withstand temperatures of up to $60^{\circ} \mathrm{C}$ for a short time.

Therefore, these constructs may have widespread applicability in the engineering of collagen-rich tissues.

Keywords: type I collagen, Langmuir Blodgett film deposition, LB, sample geometry, dipping direction, alignment distribution, pattern, tissue engineering, matrix, scaffold, coating, biomaterial.

\title{
I. Introduction
}


Collagen is the major structural protein in animals, comprising up to $30 \%$ of total protein weight. ${ }^{1,2}$ It is the foundation of the extracellular matrix in a variety of connective tissues including bone, dentin, cartilage, tendons, dermis and cornea. Individual collagen molecules are composed of three left-handed helical polypeptide chains forming a right-handed triple-helical structure that is stabilized by hydrogen bonds. This trimetric collagen, sometimes termed tropocollagen, is approximately $300 \mathrm{~nm}$ long and 1.5 $\mathrm{nm}$ in diameter. ${ }^{1,3,4}$ Tropocollagen molecules aggregate in the extracellular space in a characteristic staggered fashion to form microfibrils and fibrils. ${ }^{1}$ Bundles of fibrils form collagen fibers, a common structure in many connectives tissues. Depending on the tissue and its function, these fibers can form layers of randomly oriented molecules, they can show arrangements of high orientation in a single direction, or they may be present in multiple layers with each layer of the highly oriented fibers angled to each other. For instance, mature bone is lamellar with the adjacent lamellae differing in collagen orientation by 90 degrees; whereas, in immature woven bone, collagen is randomly oriented. ${ }^{5,6}$ Tendon collagen molecules are all oriented parallel to the long axis of the tissue, ${ }^{7}$ while cornea shows an orthogonal arrangement of aligned collagen fibrils ${ }^{8}$ and dermis a random orientation. ${ }^{9}$ These differences in orientation and structure provide the appropriate mechanical properties for the specific tissues. ${ }^{10}$

Various approaches have been used to incorporate collagen into artificial constructs for the replacement and regeneration of damaged tissues. Collagen-rich tissue, such as that from the heart valves of pig or cow, are chemically modified and used as valve replacements in human. ${ }^{11}$ Similarly, animal collagen can be extracted, purified, processed and used for a variety of purposes, including wound dressings, scaffolds, cornea/lip augmentation, enzyme immobilization, drug delivery and many other applications. ${ }^{11}$ Despite these successful applications of collagen in medicine, mimicking natural fiber arrangement and orientation is a major challenge.

Using the Langmuir-Blodgett technology, ${ }^{12}$ we have recently introduced a straight forward method to fabricate highly oriented thin film coatings of type I collagen, ${ }^{13}$ and found that the behavior of collagen molecules during film processing is comparable to a particular class of synthetic polymers known as hairy rod polymers. ${ }^{14}$ One particular similarity of both types of molecules is shape persistence. In hairy 
rod macromolecules, shape persistence is achieved by direct synthesis of a bonding pattern of the polymer chain's constitutive elements preventing the randomization of the chain trajectory as it occurs in random coiled polymers. Hairy rod macromolecules consist of a rigid backbone and a shell of flexible side groups. The side groups can be chosen as hydrophobic entities, so that a repulsive interaction, with e.g. water, can be achieved. ${ }^{14}$ As described by Wegner, ${ }^{14}$ it is relatively simple to achieve an internal nanoscale architecture within monolayer films of these macromolecules using the Langmuir-Blodgett (LB) technology. Wegner and coworkers have carried out extensive work on hydrophobic hairy rod polymers and found that LB transfer of these "hairy rods" onto plane substrate surfaces resulted in the formation of monomolecular thin films with highly-oriented molecules, preferentially aligned in dipping direction. ${ }^{14,15}$ Moreover, if annealed $\left(60-130{ }^{\circ} \mathrm{C}\right)$, layers of even higher molecular order were achieved. ${ }^{15,16,17}$ Similarly, we have demonstrated that collagen fibrillar aggregates preferentially align along to the dipping direction. ${ }^{13}$ But we have also observed that those collagen fibillar aggregates, which were initially in contact with the substrate surface before LB-transfer, were part of an "orientation arch" formed by the LB transfer near the top of the samples. Here the orientation was perpendicular to the dipping direction, parallel to the air water interface. ${ }^{13}$ The reason for this finding was suggested to be an outcome of the fabrication process. The hydrophobic substrates were mounted in the LB trough nearly completely immersed in the subphase before collagen was applied to the air-water interface. The collagen molecules on the trough, which come in close proximity to the substrate at the air-water interfacial line after spreading and compression, adhere to the substrate surface via attractive hydrophobic-hydrophobic interactions and align themselves parallel to the substrate surface and parallel to the air-water interface. The following film-lift process - the pulling-up of the substrate through the collagen LB film - results in an orientation of collagen molecules parallel to the dipping direction, an outcome driven by the moving action (substrate through the collagen film). In other words, orientation of collagen parallel to the dipping direction occurs only during slow LB transfer of the molecules onto the substrates. As mentioned above, these findings show strong similarities to observations made by Schwiegk et al. (experimentally and theoretically) for hairy rod polymers transferred to rectangular 
substrates. ${ }^{15}$ Moreover, Wegner and coworkers have shown that alignment of these molecules can be achieved in both film-transfer-directions, upstroke and downstroke. ${ }^{14,15,16}$ Schwiegk et al. ${ }^{15}$ also investigated the influence of the flow on the LB trough towards the substrate by varying the substrate width and thickness. They found that only a convergent flow leads to an alignment of the hairy rods parallel to the dipping direction. If flow was non-convergent the orientation of the rod-like molecules was perpendicular to the dipping direction.

In the present study, we have applied LB fabrication technology to deposit thin layers of collagen on flat, hydrophobic substrates with various shapes and dimensions (squares, triangles, diamonds, etc.) to arrange alignment of collagen molecules with a systematic change in orientation. We have studied effects of the sample shape, the dimension and the tilt angle with respect to the dipping direction, and have analyzed the resulting collagen orientation distribution on these $2 \mathrm{D}$ samples. In addition, we have tested the stability of these collagen films under different conditions. Optical microscopy was used to individually characterize the distribution of collagen orientation and its stability at various conditions.

\section{Experimental Section}

Collagen solution. Type I collagen with a molecular weight of $\sim 300 \mathrm{kDa}$ was prepared from rat tail tendons as previously described. ${ }^{18}$ The acid-soluble collagen received has been shown to exhibit the triple-helical structure of type I collagen (tropocollagen). ${ }^{19,20}$ For LB experiments $2 \mu \mathrm{M}$ collagen solutions were prepared by dissolving $0.6 \mathrm{mg}$ collagen in mixtures of $0.9 \mathrm{ml}$ acetic acid $(5 \mathrm{mM})$ and 0.1 $\mathrm{ml} \mathrm{n-propanol} \mathrm{(n-propanol} \mathrm{stabilizes} \mathrm{the} \mathrm{protein} \mathrm{structure} \mathrm{and} \mathrm{supports} \mathrm{the} \mathrm{film} \mathrm{formation}{ }^{13}$ ) for at least $12 \mathrm{~h}$ at $4^{\circ} \mathrm{C}$ (in motion). These solutions contain collagen still exhibiting its tropocollagen tertiary structure as shown by CD spectroscopy. ${ }^{13}$

Fabrication of hydrophobic glass substrates for LB transfer. Hydrophobization of glass surfaces was achieved by thorough cleaning and preconditioning of glass substrates followed by a self-assembly 
process of octadecyltrichlorosilane on the glass surfaces. First, for silanizing of glass substrates, creation of -OH groups on the glass surface is essential. Glass slides (Microscopy slides, Fisher Scientific) were thoroughly cleaned by sonication in $2 \%$ Hellmanex solution (Hellma, Germany), followed by a treatment in acetone and then in Milli-Q water ( $\rho \geq 18 \mathrm{M} \Omega \mathrm{cm}$, Millipore) for 5 minutes, respectively. After each step the substrates were rinsed 5 times with Milli-Q water. The slides were then immersed in a 4:1 solution of concentrated $\mathrm{H}_{2} \mathrm{SO}_{4}$ and $\mathrm{H}_{2} \mathrm{O}_{2}$ overnight, washed with copious amounts of Milli-Q water, and dried with $\mathrm{N}_{2}$. Contact angle measurements (Goniometer NRL Model 100-00, ramé-hart instrument co, Montréal) confirmed the hydrophilic nature due to creation of $-\mathrm{OH}$ groups on the slide surfaces $\left(\theta<2^{\circ}\right)$.

For the silanization step (creation of hydrophobic surfaces), a $10 \mathrm{mM}$ solution of $\mathrm{n}$ octadecyltrichlorosilane (OTS, 97\%, Sigma Aldrich) in anhydrous toluene was prepared. Since OTS is easily hydrolyzed in atmospheric moisture, this solution was always prepared under nitrogen atmosphere (using a glove box) and used immediately. Slides were immersed in OTS and given about 6 hours of OTS-assembly time in the glove box. The slides were removed from the OTS solution, rinsed with toluene and ethanol, and dried with $\mathrm{N}_{2}$. The slides were then placed in a vacuum oven at $120^{\circ} \mathrm{C}$ for one hour (20 min heating +40 min under vacuum). Contact angle measurements confirmed the hydrophobicity of the surface: $\theta>100^{\circ}$.

Langmuir-Blodgett (LB) Technique. For the LB transfer of collagen onto hydrophobic substrates, samples were immersed nearly completely in the Milli-Q water subphase. $2 \mu \mathrm{M}$ collagen solution was applied drop-wise to the entire surface of the Milli-Q water subphase in the LB trough (KSV Instruments LTD Model KSV3000-2 LB). Following a 20 minutes waiting period to allow for the volatile solvent to evaporate, either an area-pressure isotherm (surface pressure $\Pi$ vs. trough area) was obtained at a barrier compression speed of $5 \mathrm{~mm} / \mathrm{min}$ (room temperature) or the barriers were allowed to compress the collagen up to $\sim 3 / 4$ of the highest achievable pressure ${ }^{13}$ at a speed of $5 \mathrm{~mm} / \mathrm{min}$ (at room temperature). The compressed films were visible as "sticky foils". For LB film-transfer, the substrate 
was then moved out of the water subphase through the collagen LB film with a KSV film lift at a speed of $5 \mathrm{~mm} / \mathrm{min}$. For collagen deposition onto another sample, the trough was thoroughly cleaned, refilled with Milli-Q water and the new substrate mounted nearly completely immersed in the water subphase, before fresh collagen was reapplied and the fabrication process repeated.

Stability treatment of samples. To test the stability of the collagen LB films, a set of samples were treated under different environmental conditions and for various periods of time. Film stability was tested (a) at $37^{\circ} \mathrm{C}$ for 77 days in air, (b) at $37^{\circ} \mathrm{C}$ for 70 days in Ringer (buffer) solution containing (in mM) $130 \mathrm{NaCl}, 5 \mathrm{KCl}, 20 \mathrm{HEPES}, 10$ D-glucose, $1 \mathrm{MgCl}_{2}, 1 \mathrm{CaCl}_{2}$ (adjusted to pH 7.4 with $\mathrm{NaOH}$ ) and (c) under elevated temperatures at $37,45,50,55,60{ }^{\circ} \mathrm{C}\left( \pm 0.02^{\circ} \mathrm{C}\right.$ for all adjusted temperatures). For treatment (a) and (b), the samples were stored in an incubator (Baxtor) and transferred to a heated stage $\left(37^{\circ} \mathrm{C}\right)$ of a Nikon Eclipse TE 300 microscope (Nikon, Japan; temperature controller: Tempcontrol 37Analog, Zeiss, Germany) at day 0, 7, 14, 21, 28, 35, 70 and 77 to study the films. Samples in Ringer solution were dried before inspection and re-immersed after analysis. For treatment (c), annealing occurred in situ on a heating stage of the Nikon microscope for 4 min at each temperature step, while the heating time $\Delta \mathrm{t}$ between each temperature step was set to be $3.5 \mathrm{~min}$, respectively. Investigation of these samples occurred directly during the treatment.

Optical Microscopy. To analyze and study the orientation distribution of the LB collagen films on hydrophobized substrates, bright field microscopy (Zeiss Axioskop 2 MAT, Germany; Nikon Eclipse TE 300, Japan) was carried out. For some substrate surfaces, micrographs show “dotty” artifacts besides the collagen fibrillar structures. These artifacts originate from glass particles deposited during cutting the glass substrates by a glass saw. Unfortunately, these particles could not be removed.

\section{Results and Discussion}


One of the main findings of our previous studies on collagen film deposition is the formation of fibrillar aggregates of collagen (length $\leq 100 \mu \mathrm{m}$ ) embedded within the collagen matrix, both oriented in the same direction. ${ }^{13}$ These features are confirmed in the present study and used to indicate the collagen orientation. As the fibrillar aggregates are large enough to be imaged by conventional refraction limited optical microscopy, large-area analysis of orientation and orientation distribution of fibrillar collagen aggregates was carried out by bright field microscopy.

Substrates with various $2 \mathrm{D}$ shapes (squares, rectangles, triangles, disks and rhomboids) and different sizes were mounted in the LB-trough to deposit collagen. In addition, samples were mounted under various directions and angles with respect to the dipping direction. All substrates used were kept in dimensions (typically $10-12 \mathrm{~mm}$ and $25 \mathrm{~mm}$ in width) small enough to satisfy the conditions for convergent flow pattern on the trough. ${ }^{15}$

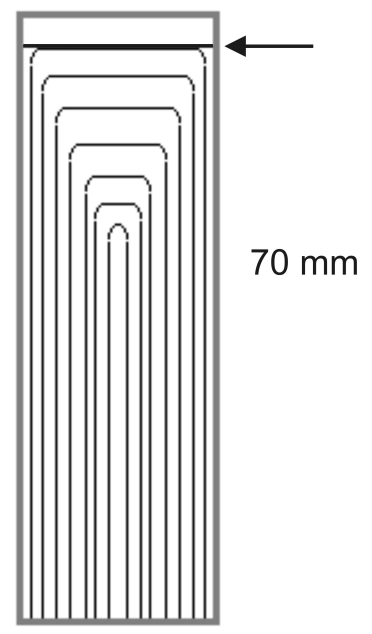

$25 \mathrm{~mm}$

Fig.1 Orientation distribution of fibrillar collagen coated at a $70 \mathrm{~mm}$ x $25 \mathrm{~mm}$ substrate. Dipping direction parallel to long axis. Arrow: air water interface before LB transfer was initiated. 
As described by Tenboll et al. ${ }^{13}$, if a rectangular substrate $(70 \mathrm{~mm}$ x $25 \mathrm{~mm})$ is mounted with its long axes parallel to the dipping direction, coating with collagen will result in fibrillar aggregates forming an "orientation arch" at the top of the sample (upper $\sim 1 / 3$ of the coated area) and to collagen alignment parallel to the dipping direction at the lower part of the sample $(\sim 2 / 3$ of the coated area). Using this substrate geometry and size, this collagen orientation distribution was reproduced several times (Fig.1).

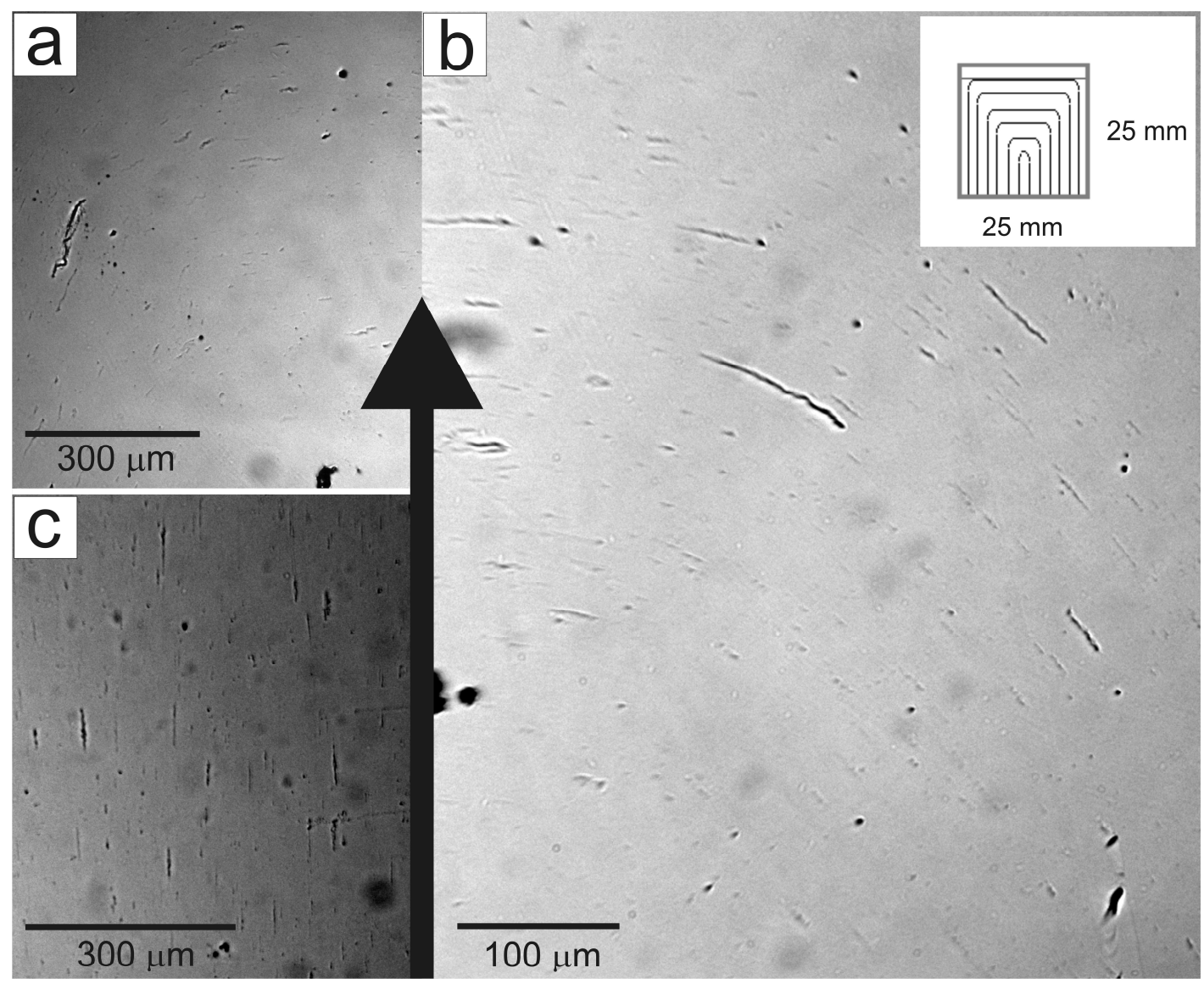

Fig.2 Bright field microscopy images taken from various areas of a collagen coated sample (25 $\mathrm{mm} \mathrm{x}$ $25 \mathrm{~mm}$ substrate): a) left hand side of arch, b) right hand side of arch (inset: sketch of the coated fibrillar orientation distribution on the square), c) lower edge (left side). 
However, the present study shows that if a square (with the same width of $25 \mathrm{~mm}$ ) is coated under identical conditions a different distribution pattern is formed. Fig.2 (inset in 2b) shows a sketch of a substrate $(25 \mathrm{~mm} \times 25 \mathrm{~mm}$ ) indicating the orientation distribution of collagen found by bright field microscopy as well as three optical micrographs depicting details of the distribution from which the sketch was drawn. In contrast to the rectangular samples (Fig.1) the arch is covering more than half of the surface area of the sample (Fig.2a,b). Alignment parallel to the dipping direction is found only for the bottom-part of the sample (Fig.2c). Clearly the length of the sample parallel to the dipping direction plays a major role in the orientation distribution of collagen, a finding related to the area available for molecules to align during flow and deposition onto the substrate. ${ }^{15}$ To additionally test these findings, we mounted the rectangular sample $(70 \mathrm{~mm} \times 25 \mathrm{~mm})$ with its long side parallel to the air-water interface (the sample in Fig.1 was turned by $90^{\circ}$ ) and deposited collagen. Analyzing the deposition pattern in the sketch of Fig.3a clearly shows that the orientation distribution of the fibrillar aggregates depends on the height of the sample. In case of Fig.1 2/3 of the lower part of the substrate surface showed collagen orientated parallel to the dipping direction, whereas in case of Fig.3a an arch regime is deposited only. But again, large areas of the substrate area indicate straight orientation of collagen. Another feature being evident from Fig.1, 2 and 3a is the alignment of the fibrillar aggregates parallel to the substrate edges. The orientation is always parallel to the sample edges with the exception of the bottom line, the tear-off edge.

To further study effects of substrate geometry and size on collagen deposition patterns we conducted several experiments using various samples and mounting angles. All shapes are shown in Fig.3 (symmetric samples) and Fig.4 (asymmetric samples). The dipping direction for all samples was always the vertical. Note: some samples, e.g. triangles, can appear more than once in the symmetric regime as they can be mounted at the tip or the base. 


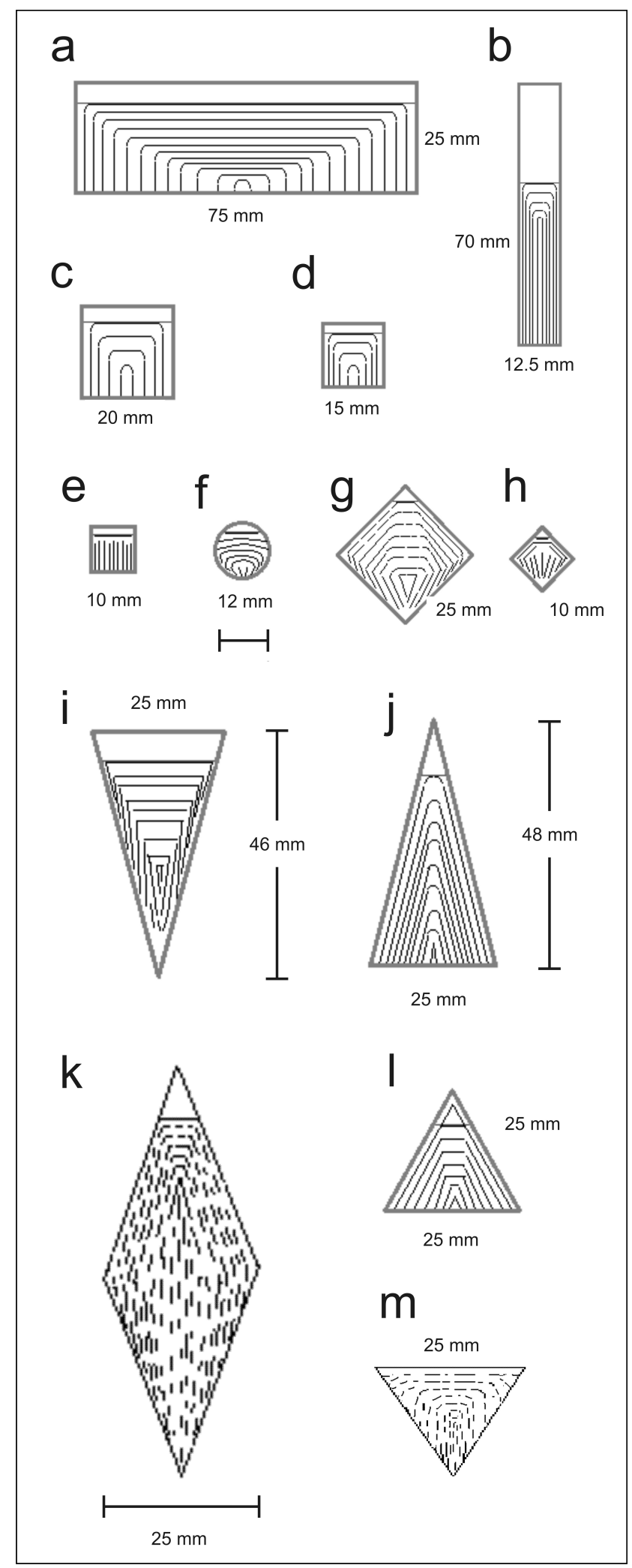

Fig.3 Sketches of fibrillar orientation distributions found on symmetric substrates (not to scale). 
For all symmetric samples (Fig.1, 2 and 3) the following orientation patterns could be observed. a) All symmetric substrates indicate the formation of a collagen "orientation arch" regardless if the top of the sample edge was straight (Fig 3a-e), round (Fig.3f) or pointy (Fig.3g-m). b) There is a clear symmetry line in the middle of each sample running parallel to the dipping direction. c) Decreasing or increasing the sample width during LB transfer (due to the substrate geometry) results in the generation of arches dominating the entire sample surface. d) For nearly all samples, collagen was aligned parallel to the sample edges when in close proximity to them (Fig.3 and 4), independent of the mounting angle. e) Triangular substrates with a tip pointing downwards during coating (angle: substrate edge/subphase $<$ 90 ; Fig.3i,m and Fig.3k [lower part]) show a preferential alignment parallel to the dipping direction, leading to an angle between the fibril alignment direction and the substrate edge. $\mathrm{f})$ Squares (25 $\mathrm{mm} \mathrm{x}$ $25 \mathrm{~mm}$ ) mounted in a tip (Fig.3g) and round samples (Fig.3f) indicate an intermediate orientation state of collagen, showing alignment which is non-parallel to the edges and non-parallel to the dipping direction. An exception to this "set of rules" was the patterning on the small squares (10 $\mathrm{mm} \times 10 \mathrm{~mm})$, when mounted at the tip or an edge (Fig.3e,h). In both cases collagen is aligning perpendicular to the dipping direction at the top of the substrate. It does, however, not form an arch, which allows collagen a smooth transition from alignment parallel (or nearly parallel) to the edges to the tear-off structure at the bottom of the samples.

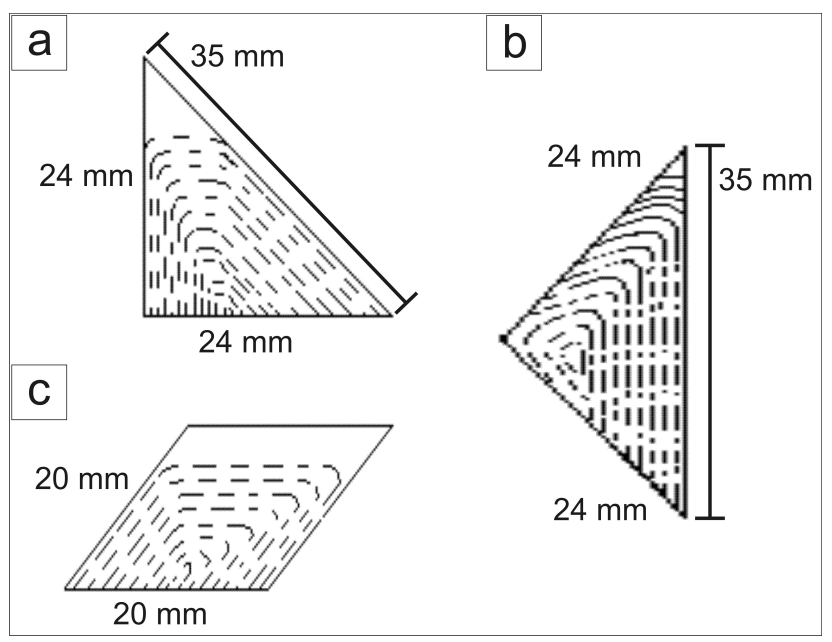

Fig.4 Sketches of fibrillar orientation distributions found on asymmetric substrates (not to scale). 
More insight into flow characteristics of collagen during film formation provides the analysis of the relationship between substrate geometries and the collagen pattern on the substrate surface. During coating of some symmetric (Fig.3j and 1 and upper halves of Fig.3g, h and k) and asymmetric samples (Fig.4) the orientation of the fibrils followed the edges of the substrates (except for the tear-off edge at the bottom), but not necessarily the dipping direction.

An explanation for this behavior is found (in part) in the flow dynamics during LB film formation investigated experimentally and theoretically by Schwiegk et al. ${ }^{15}$ They have observed that moving the substrate during LB transfer causes the molecules to flow along the water-air interface (even relatively far from the substrate) and to arrange themselves parallel to each other in a way that they finally point towards the substrate (long axis of molecules perpendicular to the sample). In the case of rectangular substrates (e.g. Fig.1, 3a,b,c), the molecules will then be pulled out (with a constant width of the substrate) and form an aligned pattern parallel to the dipping direction and parallel to the substrate's edges. ${ }^{13,15}$ If, however, geometries are chosen, where the sample width is changing during film deposition, alignments of molecules will change too. Here, the molecule flow on the trough and the final collagen pattern on the substrate are strongly influenced by the changes of the sample width and therefore by the availability of deposition area on the sample. Taking these findings into account, four different kinds of flow changes can be achieved by varying the $2 \mathrm{D}$ geometry of a substrate while pulling it out of the subphase through the collagen layer. i) Keeping the width of a sample at a fixed LB transfer position relative to the subphase (see above; e.g. Fig.1, 3a,b,c); ii) keeping the width of a substrate but changing the LB transfer position relative to the subphase (Fig.4c, parallelogram); iii) increasing the width of a sample at a fixed LB transfer position (e.g. Fig.3j,1; upper half of Fig.3g,k); and iv) decreasing the width of a sample during LB transfer at a constant position (Fig.3i and m; lower half of Fig.3f,g,h and k; lower left half Fig.4b). Note: Only those samples (or sample areas) with decreasing width during LB transfer will not show collagen aligned parallel to the edges. The edge alignment tears off; generating an "open structure" with fibrils aligned parallel/near parallel to the dipping direction. 
In general, geometries exhibiting a constant width (rectangle, square, and parallelogram) have a constant convergence in molecular flow and form (besides the arch at the top) an alignment of the collagen fibrillar aggregates parallel to the samples edges. Samples which show an increase in width (e.g. upper half of the diamond or triangle clamped at the tip) during film transfer will systematically decrease the convergence of the molecular flow on the trough during the deposition. This process results in the formation of a pronounced orientation arch and in patterns where the alignment of fibrils is parallel to the substrate edges, not parallel to the dipping direction. In contrast, sample geometries showing a decrease in width (lower half of the diamond or triangle clamped at a base) will result in an increase of convergence of the molecular flow during film formation and lead to orientation patterns where collagen is aligned parallel to the dipping direction.

Moreover, comparing the overall collagen orientation of the lower half of the long diamond shape in Fig.3k with that of the (base-mounted) triangle (Fig. $3 \mathrm{~m}$ ), both with a maximum width of $25 \mathrm{~mm}$ but different sample lengths, suggests an effect of the change in width $(\Delta \mathrm{w})$ relative to the change in height displacement $(\Delta \mathrm{h})$ via the film lift. Because, moving the sample out of the subphase by $\Delta \mathrm{h}$ results in less change in width $(\Delta \mathrm{w})$ for the long diamond (as it has a smaller tip angle) than moving the triangle with the larger tip angle of $45^{\circ}$ out of the subphase. Thus, a more distinct alignment of collagen parallel to the dipping direction is found in the large diamond (lower half) case. This effect is also observed for using squares mounted at their tips (Fig.3g and $\mathrm{h}$ ). In these cases, the change of width with $\Delta \mathrm{h}$ is similar to the base mounted triangle and leads to similar alignments: coating resulted here (Fig.3g and h; lower half and Fig. $3 \mathrm{~m}$ ) in an intermediate orientation state of collagen, showing non-parallelism of alignment to either edges and to the dipping direction. Therefore, the angel between the sample-edge and the subphase plays an important role in controlling the molecular flow, the degree of convergence, and therefore the patterning of 2D substrates with collagen fibrils.

The asymmetric sample in Fig.4b contains all $\Delta \mathrm{w} / \Delta \mathrm{h}$ variations discussed. It therefore shows all orientation distributions of the collagen fibrillar aggregates possible in a mixed fashion: 1) the pronounced arch, here from top to down, in the upper half of the sample; 2) the parallel orientation 
along the right edge and (here) along the dipping direction, because on this edge the convergence did not change; and 3) an edge alignment with an open tearing off structure along the left side, where fibrils are aligned neither parallel to the edge nor parallel to the dipping direction in the upper half of the sample and in parallel/near parallel to the edge in the lower half of the sample.
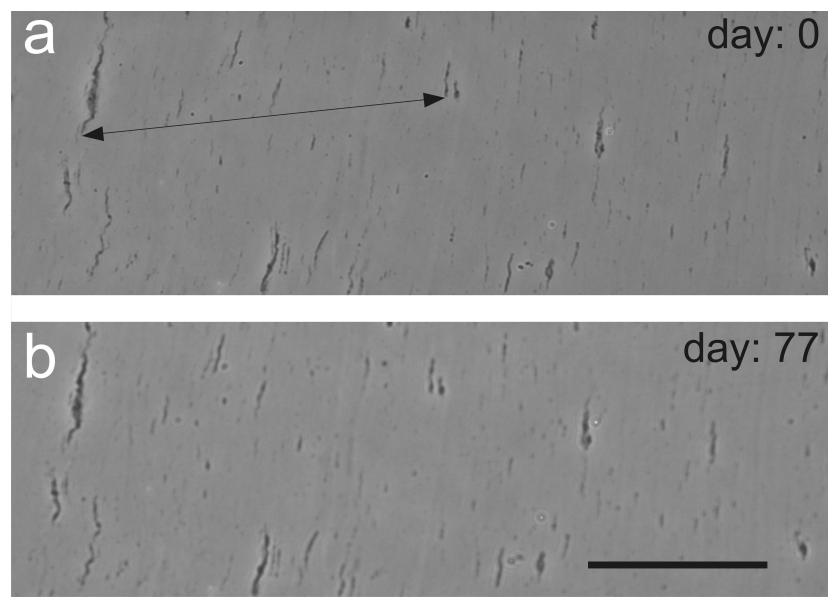

Fig.5 Bright field optical microscopy images of a sample kept in air at $37^{\circ} \mathrm{C}$ for 77 days: a) freshly prepared and b) after 77 days. The arrow shows the distance evaluated. Scale bar: $100 \mu \mathrm{m}$

For biomedical applications it is important to use substrates, which are stable with respect to a multitude of environmental effects and for a long period of time. We have, therefore, tested the stability of LB deposited collagen samples under various conditions for up to three months: a) storage in air at $37^{\circ} \mathrm{C}$ (for 3 months), b) storage in buffer (Ringer) solution at $37^{\circ} \mathrm{C}$ (for 3 months) and c) treatment in air at increasing temperature $\left(37^{\circ}-60^{\circ} \mathrm{C}\right)$. Using an optical microscope, film stability was analyzed, under the same conditions and at the same spot every week (or at a given temperature) and micrographs taken. For quantitative investigations two large fibrils were randomly chosen on each sample at the beginning of the study and the distances between them measured for each time/temperature series. Fig.5 shows images taken by bright field microscopy of a sample kept in air at $37^{\circ}$ (a) directly after preparation $(0$ days) and (b) after 77 days. The arrow in Fig.5a indicates the distance between two fibrillar aggregates, a measure also used to evaluate the film stability. In Fig.6 microscopy images of a sample stored in 
buffer solution [a) 0 days, b) 7 days, c) 70 days] at $37^{\circ}$ are shown. Again, an arrow indicates the measured distance of fibrils for evaluation of film stability.

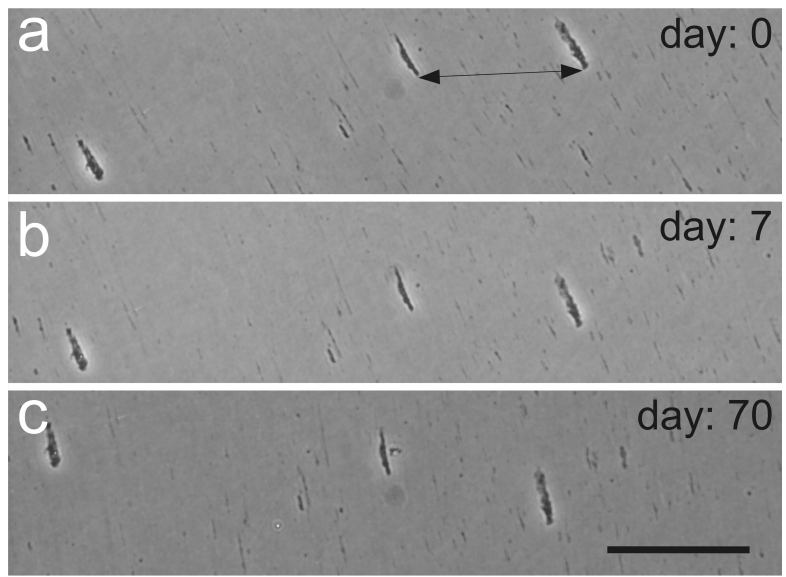

Fig.6 Bright field optical microscopy images of a sample kept in buffer solution at $37^{\circ} \mathrm{C}$ for 77 days: a) freshly prepared and b) after 7 days and c) after 70 days. The arrow shows the distance evaluated. Scale bar: $100 \mu \mathrm{m}$

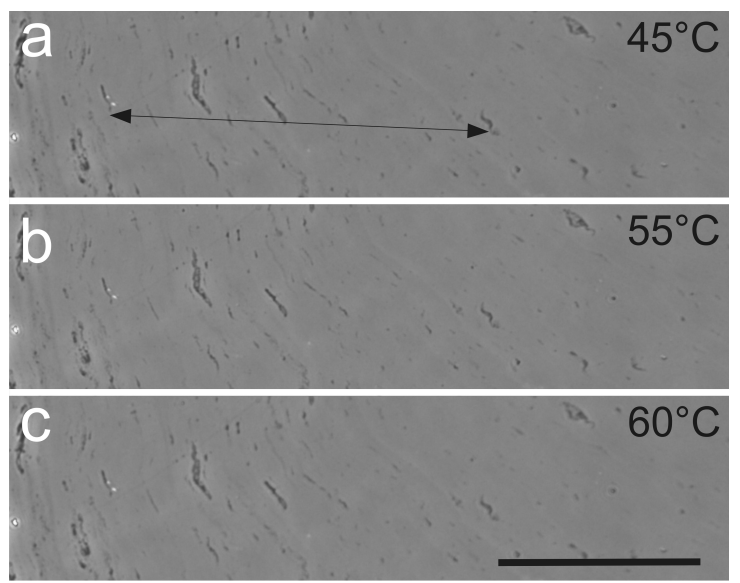

Fig.7 Bright field optical microscopy images of a sample treated by increased temperature. a) after treatment at: $45^{\circ} \mathrm{C}$, b) $55^{\circ} \mathrm{C}$ and c) $60^{\circ} \mathrm{C}$. The arrow shows the distance evaluated. Scale bar: 100 $\mu \mathrm{m}$

Images for analyses and quantification of temperature stability of collagen films are shown in Fig.7. For details, quantification of distance data (film stability) for all samples during the complete testing 
period/temperature is shown in Fig.8. The data indicate that for any sample evaluated no significant changes in measured distances were determined.

We therefore conclude that the LB deposited collagen films are stable under these three tested conditions. However, as only the position of the fibrillar aggregates has been determined, it is not clear at this point whether the collagen matrix has not undergone an orientation change during the storage or heat treatment. On the other hand, Cha et al. ${ }^{17}$ have shown that annealing of LB samples of hairy rod macromolecules (poly[bis $(m$-butoxyphenyl)silane $])$ up to $130^{\circ}$ enhances the orientation alignment, enhances the order parameter, respectively. This is a hint that the orientation direction of the collagen matrix might not have changed, however the order might have even increased. To clarify this matrix stability issue, additional studies are necessary. Thin film methods investigating the matrix' anisotropy locally can to be employed; e.g. assessing the anisotropic refractive index with localized versions of ellipsometry $^{21}$, waveguide spectroscopy ${ }^{22}$, or absorption spectroscopy as was carried out previously on these films for anisotropy studies. ${ }^{13,17}$ These tests will offer the prospect that shelf-life times of three months (at least) are achievable, and that short time exposure to temperatures of up to $60^{\circ}$ are of no concern.

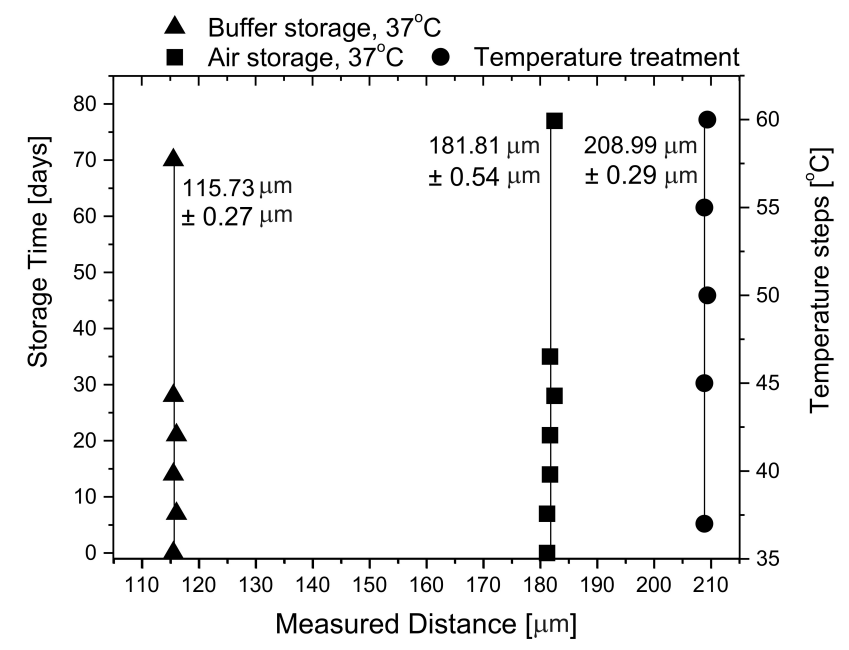

Fig.8 Measured distances versus the storage time or the temperature. The errors are within the range of the symbols. The lines represent the average distance and serve as a guide to the eye. 


\section{Conclusion}

We found that the LB-transfer of tropocollagen onto $2 \mathrm{D}$ substrates and its final orientation distribution is controlled by the particular flow conditions on the trough, the degree of convergence or divergence, directly controlled by the substrate geometry. It was demonstrated that, by choosing a particular substrate shape, a particular collagen orientation and orientation distribution can be achieved.

We also have shown that the fibrillar aggregate structure of the LB films is stable for at least 3 months and that those films can be treated at temperatures of up to $60^{\circ} \mathrm{C}$ for a short time. The orientation behavior of the collagen matrix during storage and heat treatment is very likely stable as well, however this needs to be confirmed by additional investigations.

We suggest that the 2D orientation behavior found in the present study for collagen and the possibility to control orientation and orientation distributions are not unique to tropocollagen. We assume hairy rod polymers and other macromolecules, synthetic and natural, with a long persistence length will show a similar behavior.

In a next step, a cleavable site should be introduced between the substrate surface and the hydrophobic (functionalized) surface layer allowing for a nondestructive peel-off process of the collagen layer. In this way, thin, flexible collagen films would be available for applications where certain collagen orientations and distributions are needed, e.g. for medical purposes like wound dressing, or physical investigations such as rheological studies. The orientation distribution on 3D samples, as e.g. for small implants or biomedical scaffolds, will be tested in the future.

\section{Acknowledgements.}

The authors thank the glassblower of The University of Western Ontario (UWO), Yves Rambour, for cutting the glass into the various geometries and the Nanofabrication Facility at The University of Western Ontario for hosting our LB trough. We also thank Stephen Sims (Department of Physiology \& Pharmacology; UWO) for providing the Nikon microscopy set-up. These studies were funded by NSERC and CIHR. S.M. thanks the Canadian Government for the CRC program. 


\section{References}

(1) Nimni, M. E., Ed.; Collagen Volume I Biochemistry; CRC Press: Boca Raton, 1988.

(2) Shoulders, M. D., Raines, R. T. Collagen structure and stability. Annual Reviews in Biochemistry 2009, 78, 929-958.

(3) Hulmes, D. J. Building collagen molecules, fibrils, and suprafibrillar structures, Journal of Structural Biology 2002, 137, 2-10.

(4) Hulmes, D. J. The collagen superfamily - diverse structures and assemblies. Essays in Biochemistry 1992, 27, 49-67.

(5) Currey, J. D. Bones: Structure and Mechanics; Princeton University Press: Princeton, 2006.

(6) An, Y. A., Draughn, R. A., Eds.; Mechanical Testing of Bone and Bone-Implant Interface; CRC Press: Boca Raton, 2000.

(7) Squier, C. A., Bausch, W. H., 3-dimensional organization of fibroblasts and collagen fibrils in rat tail tendon. Cell and Tissue Research 1984, 238, 319-327.

(8) Meek, K. M., Corneal collagen-its role in maintaining corneal shape and transparency. Biophysical Reviews 2009, 1, 83-93.

(9) Gross, J., Schmitt, F. O. The structure of human skin collagen as studied with the electon microscope. The Journal of Experimental Medicine 1948, 88, 555-567.

(10) Purslow, P. P., Wess, T. J., Hukins, D. W. L. Collagen orientation and molecular spacing during creep and stress-relaxation in soft connective tissues. The Journal of Experimental Biology 1998, 201, $135-140$.

(11) Meena, C., Mengi, S. A., Deshpande, S. G. Proceedings of the Indian Academy of Sciences Chemical Sciences 1999, 111 (2), 319-329.

(12) Petty, M. C. Langmuir-Blodgett films: an Introduction; Cambridge University Press: Cambridge 1996. 
(13) Tenboll, A., Darvish, B., Hou, W., Duwez, A.-S., Dixon, S.J., Goldberg, H.A., Grohe, B., Mittler,

S. Controlled deposition of highly oriented type I collagen mimicking in vivo collagen structures. Langmuir 2010, 26 (16), 12165-12172.

(14) Wegner, G. Nanocomposites of hairy-rod macromolecules: Concepts, constructs, and materials. Macromolecular Chemistry and Physics 2003, 204, 347-357.

(15) Schwiegk, S., Vahlenkamp, T., Xu , Y.Z., Wegner, G. Origin of Orientation phenomena observed in layered Langmuir-Blodgett structures of hairy-rod polymers. Macromolecules 1992, 25 (9), 2513 2525.

(16) Mittler-Neher, S., Neher, D., Stegeman, G.I., Embs, F.E., and Wegner, G. Polarization dependent resonant THG on Langmuir-Blodgett multilayers of rod-like polysilanes during annealing. Chemical Physics 1992, 161, 289-297.

(17) Cha, M., Neher, D., Mittler-Neher, S., Stegeman, G.I., Determination of the two first nontrivial orientational order parameters in LB films of rod-like molecules by $3^{\text {rd }}$-order sum frequency mixing. Chemical Physics Letters 1993, 202, 44-50.

(18) Hunter, G. K., Poitras, M. S., Underhill, T. M., Grynpas, M. D., Goldberg, H. A. Induction of collagen mineralization by a bone sialoprotein-decorin chimeric protein. Journal of Biomedical Materials Research 2001, 55, 496-502.

(19) Baht, G. S., Hunter, G. K., Goldberg, H. A. Bone sialoprotein-collagen interaction promotes hydroxyapatite nucleation. Matrix Biology 2008, 27, 600-608.

(20) Baht, G. S. Effect of collagen-binding and phosphorylation in BSP-mediated hydroxyapatite nucleation; PhD Thesis, Biochemistry, The University of Western Ontario: London, ON, 2009.

(21) Gonella, G., Cavalleri, O., Emilianov, I., Mattera, L., Canepa, M., Rolandi, R., Spectroellipsometry on cadmium stearate Langmuir-Blodgett films. Materials Science and Engineering C Biomimetic and Supramolecular Structures 2002 22(2), 359-366. 
(22) Mittler-Neher,S., Otomo, A., Stegeman, G.I., Mehta, R., Agrawal, A.K., Jenekhe, S.A., Waveguiding in substrate supported and free standing films of insoluble conjugated polymers. Applied Physics Letters 1993, 62(2),115-117. 
TOC Graphic- table of contents only

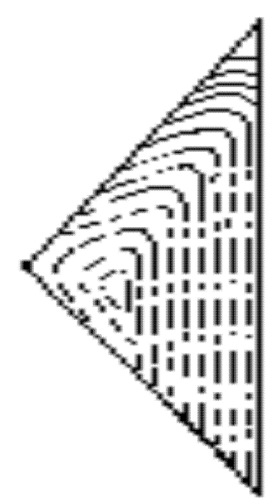

